\title{
Mini-commentary on BJOG-19-1671.R2
}

\author{
Herbert Valensise ${ }^{1}$ \\ ${ }^{1}$ University of Rome Tor Vergata
}

May 6, 2020

The interesting article published on this issue by Helen Perry et al (BJOG $2020 \mathrm{xxxx}$ ) is an important contribution to the idea demonstrated several years ago with echocardiographic evaluation of maternal central parameters, that the blood pressure value alone is not useful to understand the degree of compromise of the maternal condition in the evaluation of gestational hypertension and Preeclampsia (Valensise $\mathrm{H}$ et al. BJOG. 2006 Sep;113(9):1044-52. Epub 2006 Jul 7). The authors present an elegant study in which the evaluation of the maternal cardiovascular parameters obtained through non-invasive methods (Continuous Waveform Doppler USCOM) show major differences in cardiac output, systemic vascular resistances and heart rate in those patients with the diagnosis of Preeclampsia with a reduced fetal growth.

These findings open two lines of research that needs to be implemented on the future: the first is linked to the possibility to identify 'personal targeted therapy' according to the parameters found to ameliorate the placental perfusion and fetal growth increasing the heart rate when below the normal values, reducing the systemic vascular resistances and increasing the plasma volume so as to try to bring back to normal the cardiocirculatory conditions (Valensise H et al. Ultrasound Obstet Gynecol. 2008 Jan;31(1):55-64).

The second most important research line has to concentrate to the maternal circulatory condition before pregnancy (Vasapollo B et al. Am J Obstet Gynecol. 2020 Mar 3. pii: S0002-9378(20)30234-9. doi: 10.1016/j.ajog.2020.02.043) that in high risk pregnancies might induce not only an automatic passive calculation of the risk factors, but an active therapy based on the modifications of the maternal cardiac parameters to bring them back to normal as much as possible to PREVENT the appearance of the abnormal adaptation to pregnancy.

In the future maternal health control in pregnancy will not be possible without the knowledge of maternal cardiac hemodynamics before pregnancy, in the first and second trimester, and at the appearance of hypertension or signs of fetal growth restriction. For this reason the implementation of clinical research with the use of non-invasive devices accessible to any doctor need to be recommended.

No disclosures: A completed disclosure of interest form is available to view online as supporting information. 